\author{
K. Ciecieląg ${ }^{1 *}$, K. Kęcik² ${ }^{2}$ K. Zaleski ${ }^{1}$ \\ ${ }^{1}$ Lublin University of Technology, Mechanical Engineering Faculty, Department of \\ Production Engineering, Lublin, Poland \\ ${ }^{2}$ Lublin University of Technology, Mechanical Engineering Faculty, Department of Applied \\ Mechanics, Lublin, Poland \\ *k.ciecielag@pollub.pl
}

\title{
EFFECT OF DEPTH SURFACE DEFECTS IN CARBON FIBRE REINFORCED COMPOSITE MATERIAL ON THE SELECTED RECURRENCE QUANTIFICATIONS
}

\begin{abstract}
The paper discusses the problem of possibility of the detecting surface defects in carbon fibre reinforced plastics (CFRP) materials on the basis of the milling time series. First, the special defects in the hole-shaped with various depth were made. Next, the cutting forces are measured during the milling machining. Finally, the recurrence plot and quantification analysis was applied. The obtained results show that the depth defect influences the selected recurrence quantifications, which can be used as the simple defect indicators. The conducted research allow to determine the percentage share of the detectable defects. The novelty of the work and an unresolved problem is the selection of recurrence quantifications with the simultaneous use of them to detect the size of defects in carbon fibre reinforced plastics.
\end{abstract}

Keywords: recurrence quantifications; recurrence plots; carbon fibre reinforced plastics; milling

\section{INTRODUCTION}

Non-linear analyzes are a modern approach to research of machining. One of the latest methods of non-linear signal analysis are recurrence methods (RP). Currently, they are very widespread in various areas of activity. They find application in industry $[1,2,3,4,5]$ as well as in quite different areas, such as economics [6]. Non-linear analysis can detect damage at the stage of their formation. The development of the RP allows their application in medicine [7] in the field of research of heart rhythm. By analyzing heart rate, repeatability and cyclicity of strokes, you can determine the initial symptoms of the disorder in his work. Based on recurrence methods, they can be used for research of urban traffic [8], where sensors can be used to check mass and overload of moving vehicles. Due to unfavorable climatic changes, non-linear data analysis methods can be successfully used to "predict" upcoming weather anomalies [9]. On the basis of time series, it has been proved that it is possible to use RP and recurrence quantifications (RQA) to detect defects [10].

Cognitive capabilities of people in the field of nonlinear studies have been extended with the development of computer techniques. In 1987, Jean-Pierre Eckmann introduced a recurrence plot to the non-linear data analysis [11]. Creating a recurrence plot is based on 
the delay coordinates and the phase space reconstruction. In the delay coordinates in the $m$-dimensional space a reconstructed vector is created. Reconstruction is related to the selection of the embedding delay $d$, the embedding dimension $m$ and the cut-off distance $\varepsilon$ (threshold). The reconstructed vector can be represented by the equation (1):

$$
\boldsymbol{x}=\left(x_{i}, x_{i+d}, x_{i+2 d}, \ldots, x_{i+(m-1) d}\right),
$$

where: $x_{i}$ means the $i$-th coordinate in a time course $x(t)$, the symbol $m$ represents the embedding dimension and the symbol $d$ denotes the time delay. The embedding delay can be selected using two methods. In the first method, which is used for linear systems [12], the $C(d)$ autocorrelation function can be used:

$$
C(d)=\frac{1}{N-d} \sum_{i=1}^{N-d}\left(x_{i}-\bar{x}\right)\left(x_{i+d}-\bar{x}\right)
$$

where: $x_{i}$ means the $i$-th coordinate in a time course $x(t), \bar{x}$ means the average value of all coordinates, and $N$ is the number of measuring points. The second method is called the average mutual information function (AMI), which aims to determine the averaged amount of information in one state, which may be the result of prediction from information that was in a previous state:

$$
A M I=-\sum_{i j} p_{i j}(d) \ln \frac{p_{i j}(d)}{p_{i} p_{j}},
$$

where: $p_{i j}$ means the probability that the observation of a specific moment of time $t$ belong to $i$-th section, and $p_{i}$ determines the probability of finding the time value of the system in the $i$-th section. The place of the first minimum of the AMI function is the embedding delay $d$ [13]. The choice of dimension $d$ is the first stage needed to reconstruct the vector. The signal should be chosen accordingly, because too high value will cause interference, and too small value may cause illegibility of recurrence plots and recurrence quantifications.

The choice of the embedding dimension $m$, carried out using the method of false nearest neighbors (FNN), should not take into account false points on the recurrent plot, which may influence on the structure of the plot [14]. The value of the cut-off parameter $\varepsilon$ is significant due to the number of points obtained on the recurrence plot, because too small a value $\varepsilon$ can cause that the plot will be useless and there will not be any recurrence points [15].

Recurrence plots provide only qualitative information, so Zbilut and Webber $[16,17]$ and Marwan [18] introduced the recurrence quantifications. Among the large number of quantifications, we can distinguish quantifications such as: recurrence rate $(R R)$, determinism $(D E T)$, average diagonal length $(L)$, length of the longest diagonal line $\left(L_{\max }\right)$, entropy $(E N T R)$, laminarity $(L A M)$, trapping time $(T T)$, the longest vertical line $\left(V_{\max }\right)$, recurrence time of the $1^{\text {st }}$ and $2^{\text {nd }}$ type $(T 1, T 2)$, recurrence period density entropy (RPDE), clustering coefficient $(C C)$ and transitivity (TRANS) [19, 20, 21]. The definition of all recurrence quantification can be found in papers [18]. Generally, we have two basic approaches by means of which recurrence quantifications can be used. The first method assumes that the cutoff parameter $\varepsilon$ is constant and the second the RR measure is constant.

The motivation for writing of this paper is to find the new method for detection of defects in the composite material. Usually, in the composite materials defects are detected by scanning methods. However, this method is useless if defects arise during cutting. Then the recurrence methods can be applied.

In literature we can find papers confirm that recurrence methods are able to detect defects [22-24]. However, is lack of papers which analyze the problem of the size of the detectable defects. The novelty of this paper is determination of the smallest defect possible to detect. 


\section{METHODOLOGY}

Carbon fibre reinforced plastics were in the form of plates consisting of 50 alternately arranged prepregs (system $0-90^{\circ}$ ). The panels with dimensions of $300 \mathrm{~mm}$ x $300 \mathrm{~mm}$ x $15 \mathrm{~mm}$ were made in special rooms while maintaining the temperature conditions (temperature was in the range of $18^{\circ} \mathrm{C}$ to $30^{\circ} \mathrm{C}$ ) and adequate humidity not exceeding $60 \%$. The room cleanliness necessary to produce composite materials should not exceed 10,000 particles per $1 \mathrm{~m}^{3}$. After heating the material in an autoclave for 2 hours at $177^{\circ} \mathrm{C}\left(+/-2^{\circ} \mathrm{C}\right)$ and pressure of $0.3 \mathrm{MPa}$, they were subjected to finishing operations.

Prepared samples after heating in the autoclave and cooling in the ambient atmosphere were intended for further machining. The tools dedicated to the machining of carbon fibre reinforced plastics saturated with epoxy resin were Kennametal cutters (symbol 20A02R028A20ED10) with interchangeable inserts (symbol EDCT10T304PDFR-PCD) with a polycrystalline diamond coating (symbol KD1410). The cutter had a diameter $d_{c}=20 \mathrm{~mm}$. During milling at the vertical machining center (Avia - VMC $800 \mathrm{HS}$ ) the feed force (accuracy: $0.000001 \mathrm{~N}$ ) were measured using Kistler 3D dynamometer (type 9257B) with the Kistler type 5070 amplifier, Dynoware data acquisition card type 5697A and DynoWare software type $2825 \mathrm{~A}$. The feed force were used to analyze recurrence plots and recurrence quantifications. The photo of milling test and the scheme of recurrence method was showed in Figure $1 \mathrm{a}$ and $1 \mathrm{~b}$.
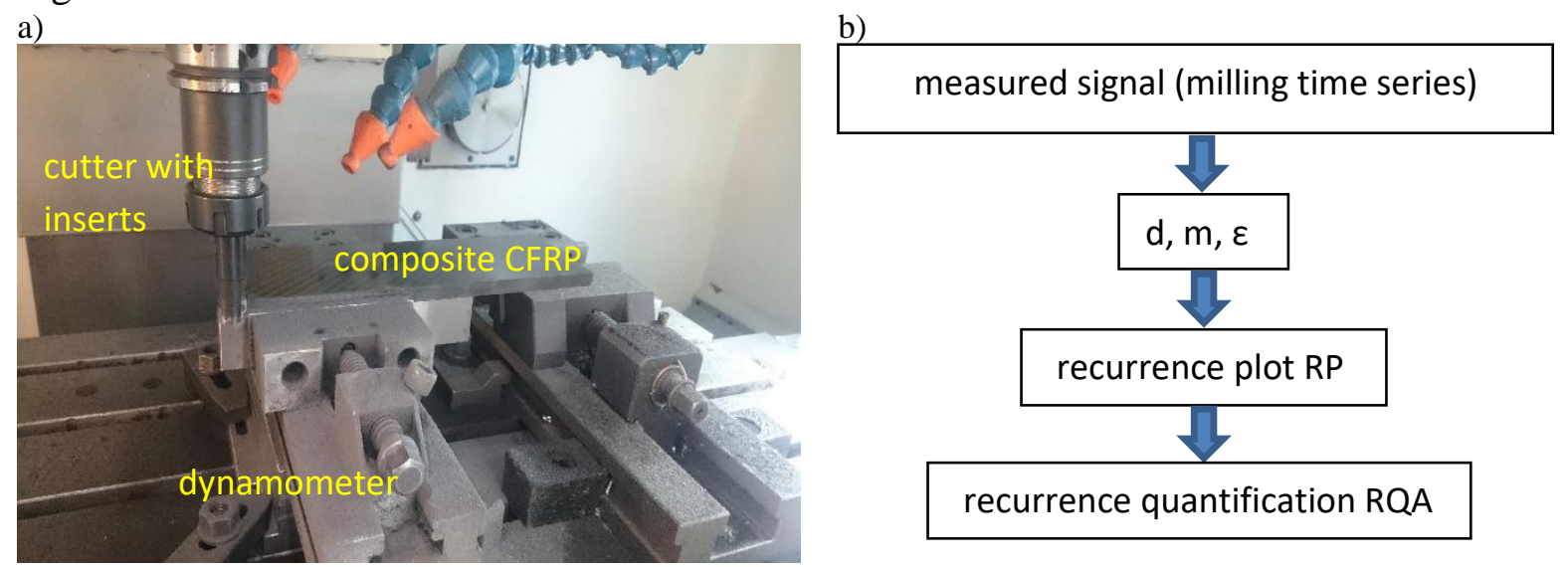

Fig. 1. Photo of milling test (a) and the scheme of recurrence methodology (b)

On the basis of the measured force values were determined of the embedding parameters: $d, m$ and threshold $\varepsilon$. The embedding delay $d$ is estimated from the AMI function. The embedding dimension $m$ is estimated using the FNN method. Next the recurrence plot has been constructed:

$$
R_{i, j}=H\left(\varepsilon-\left\|x_{i}-x_{j}\right\|\right)
$$

where $H$ is the Heaviside function, $\varepsilon$ is the threshold parameter and $x_{i}, x_{j}$ is $i, j$-th coordinate at given time course.

Last step is the recurrence quantifications analysis which is statistical analysis of the recurrence structures from the RP plot. Recurrence quantification is description of statistics of the RP plot based on the line structures and recurrence points density.

The milling tests have been performed constant cutting speed $v_{c}=250 \mathrm{~m} / \mathrm{min}$, feed $\mathrm{f}_{\mathrm{z}}=0.2 \mathrm{~mm} / \mathrm{edge}$ and depth of cut $\mathrm{a}_{\mathrm{p}}=2 \mathrm{~mm}$. The defects (holes) of various diameters $(b) \phi 2$ 
$\mathrm{mm}, \phi 4 \mathrm{~mm}, \phi 6 \mathrm{~mm}$ and various depths $(\mathrm{g}) 0.5 \mathrm{~mm}, 1 \mathrm{~mm}, 1.5 \mathrm{~mm}$ was presented in Figure 2 .

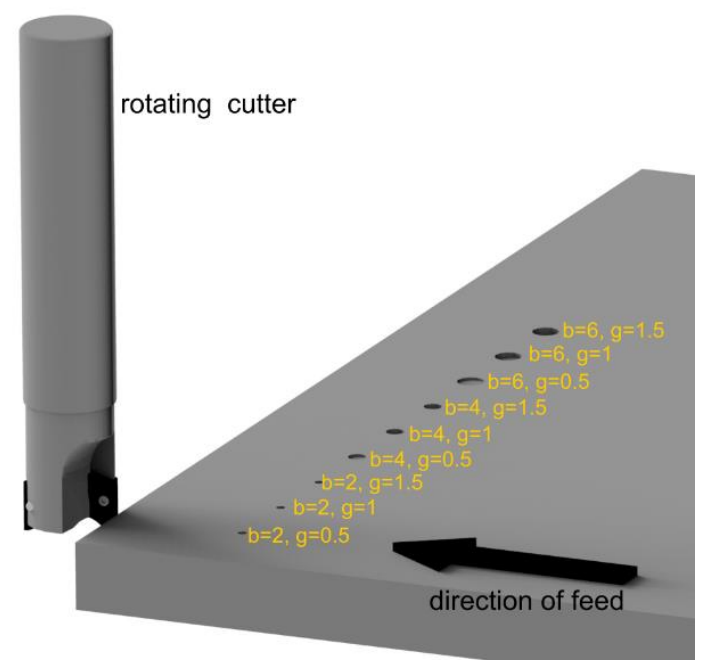

Fig. 2. The scheme of milling of carbon-fiber-reinforced plastics

The values of feed forces were used for recurrence analyzes because the registered forces during milling of surface with artificial defects were compared with the values of forces during milling of surfaces without artificial defects.

\section{RESEARCH RESULTS BY RECURRENCE METHODS}

The present study was carried out with the assumption that the recurrence rate is constant $\mathrm{RR}=0.20$ (the same number of points were analyzed for each tests). In Figure $3 \mathrm{a}$ and $3 \mathrm{~b}$ the dashed lines show the defect (the diameter of hole was $\phi 2 \mathrm{~mm}$ ) position respectively for the hole with the depth of $0.5 \mathrm{~mm}$ and the hole with the depth of $1.5 \mathrm{~mm}$. In Figure $3 \mathrm{c}$ was showed recurrence plot for surface without the hole.

a)

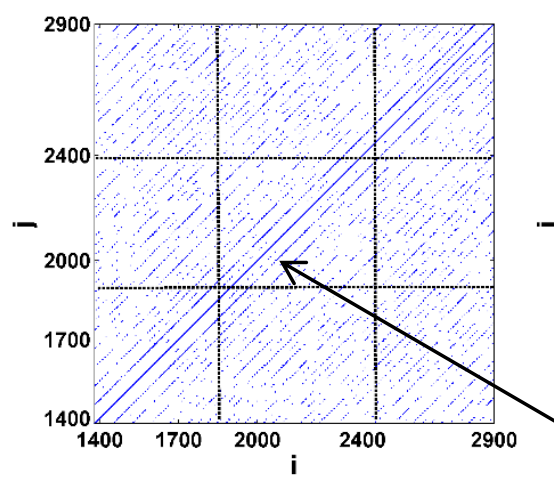

b)

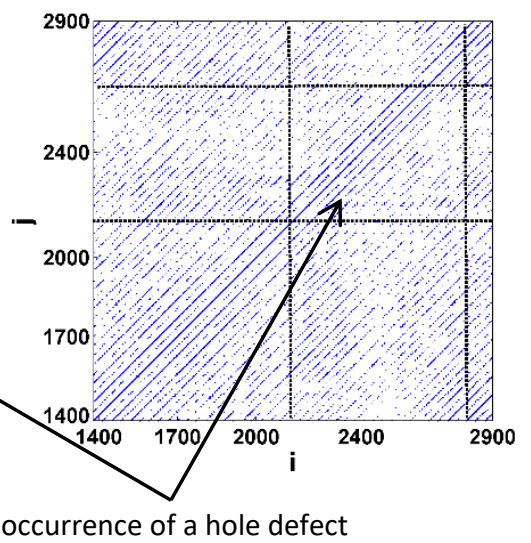

c)

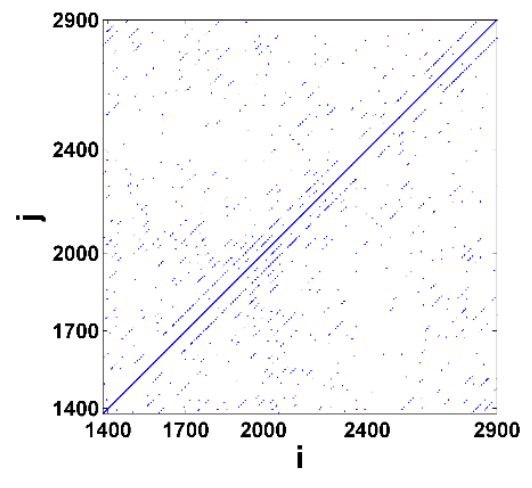

Fig. 3. Recurrence plot created as a result of surface milling: a) with the hole with the depth of $0.5 \mathrm{~mm}(d=9, m=5, \varepsilon=20)$, b) with the hole with the depth of $1.5 \mathrm{~mm}(d=8, m=5, \varepsilon=20)$,

c) and without the hole $(d=8, m=5, \varepsilon=20)$. The diameter of all hole was fixed of $\phi 2 \mathrm{~mm}$ 
The extension of the RP method is recurrence quantifications analysis. The recurrence measures quantitatively determine structures of the recurrence plots presenting the number and duration of the recurrences of a dynamical system.

In this paper only few recurrence quantifications are presented. These recurrence measures can detect the damage correctly. These indicators are: TT (Figure 4), L (Figure 5), LAM (Figure 6), T2 (Figure 7) and CC (Figure 8). The blue color line presents the RQA result for the defect with the depth of $0.5 \mathrm{~mm}$, the black color line shows RQA measures for the surfaces defect with the depth of $1.5 \mathrm{~mm}$, and the red color line means RQA indicators without defect. The vertical lines indicate the position of the defect (blue indicates the defect with the depth of $0.5 \mathrm{~mm}$, and black indicates the position of the defect with the depth of $1.5 \mathrm{~mm}$ ).

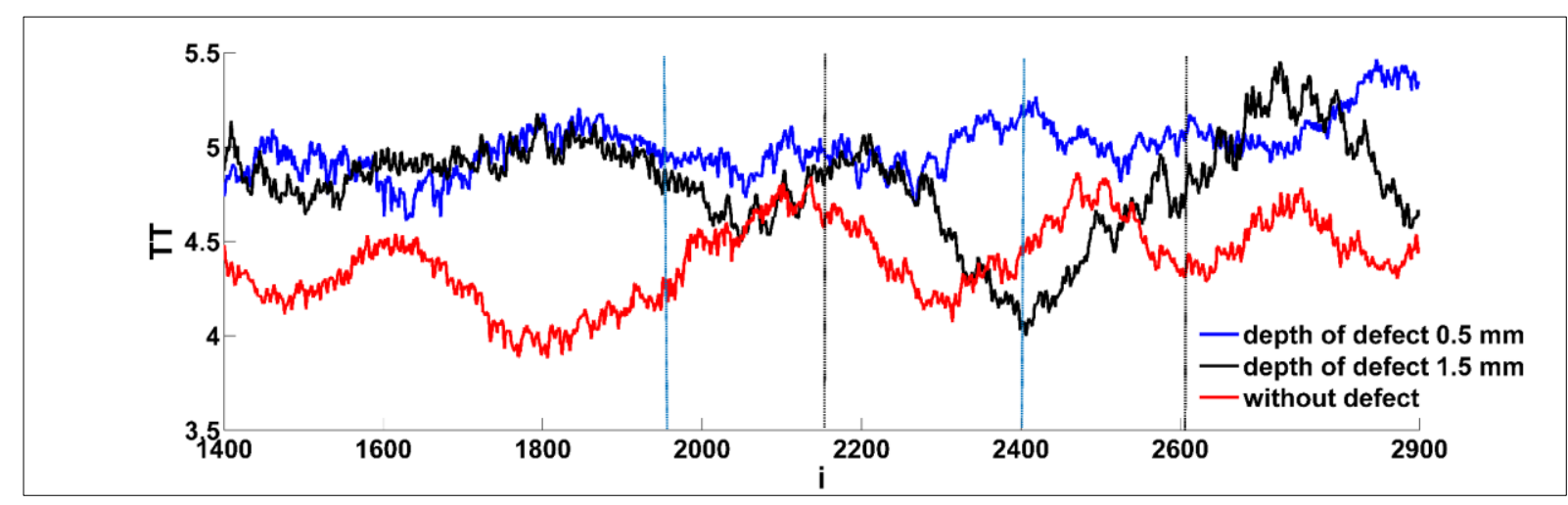

Fig. 4. Plot of recurrence quantification - TT

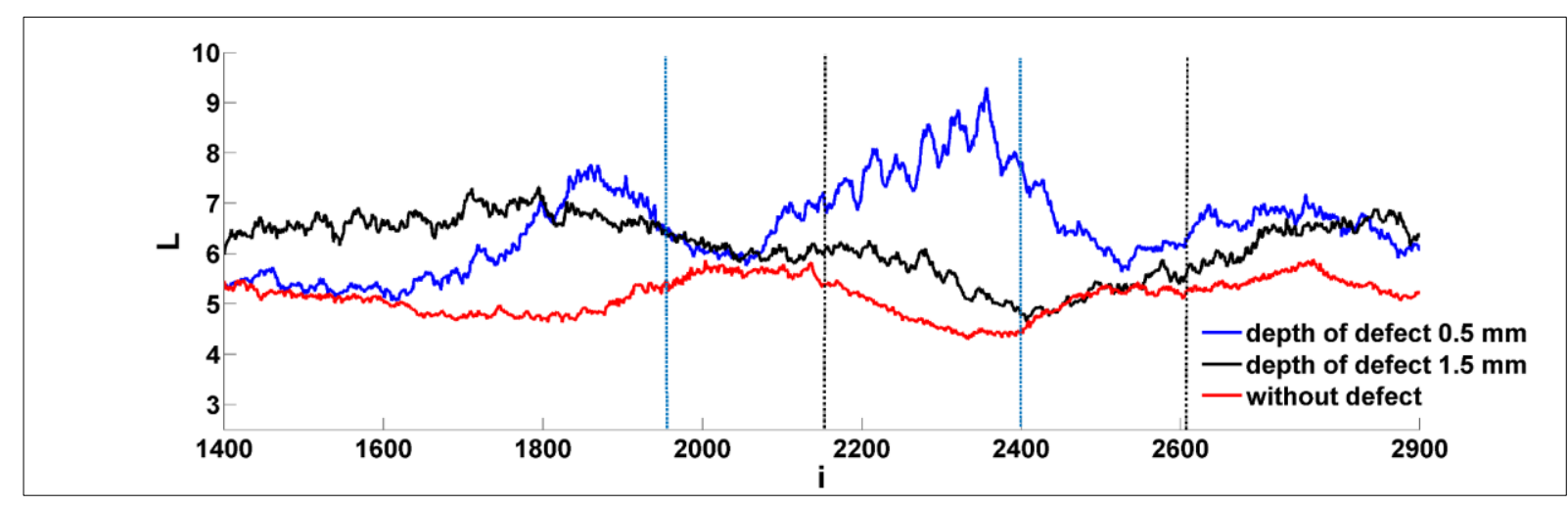

Fig. 5. Plot of recurrence quantification $-\mathrm{L}$

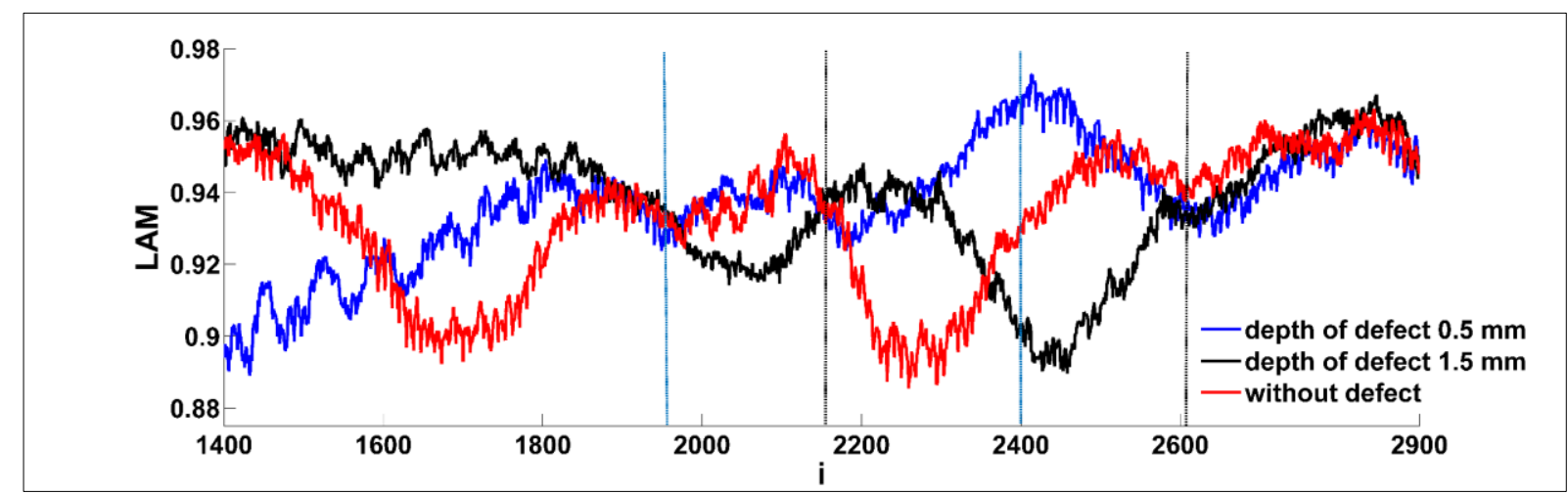

Fig. 6. Plot of recurrence quantification - LAM 


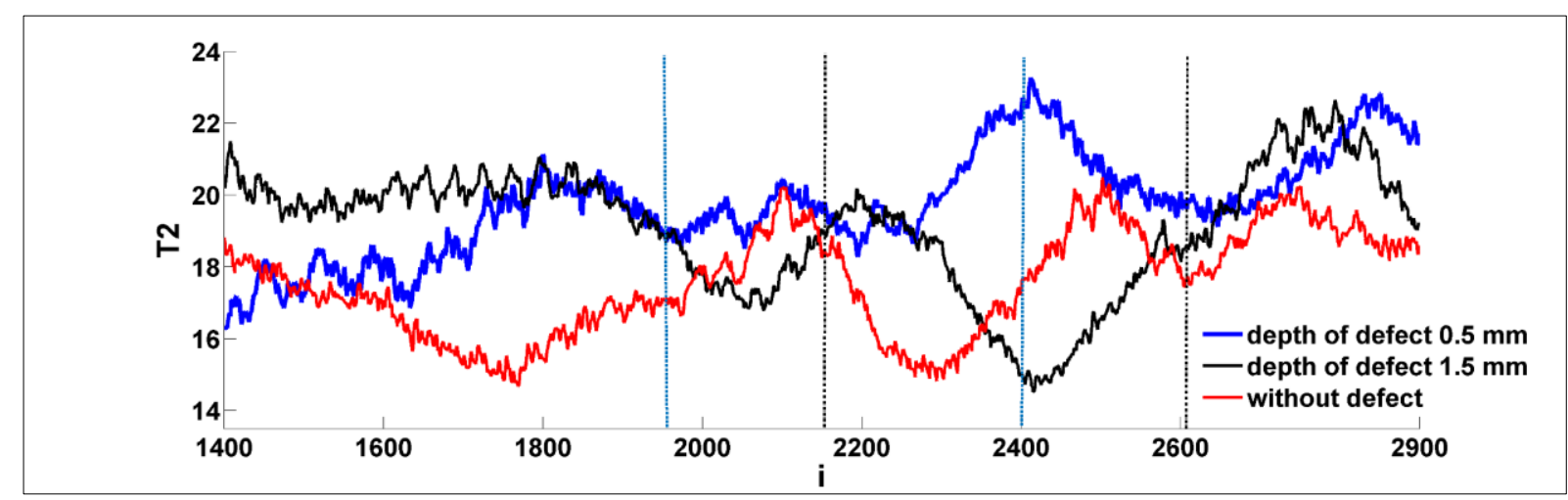

Fig. 7. Plot of recurrence quantification - T2

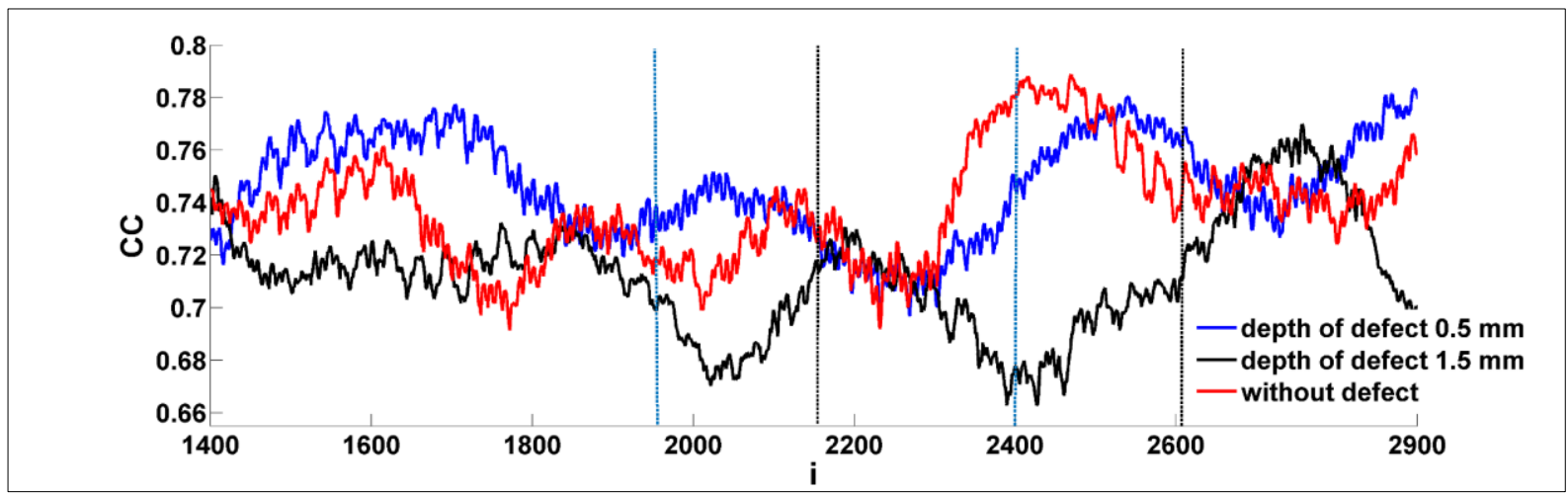

Fig. 8. Plot of recurrence quantification - CC

Figure 4 shows the plot of the quantification TT, where a drop of $20 \%$ is noticeable at the location of the $1.5 \mathrm{~mm}$ deep defect. In the plot of the quantification L shows in Figure 5 in the range 2150-2600, a decrease in the value of L to value of 5. The quantifications LAM, T2 and $\mathrm{CC}$ are shown in figures 6,7 and 8 respectively. These indicators also reduce their values in the defect position (black color vertical lines). On the basis of the RQA results, it can be concluded that it is possible to detect surface defects with a depth less than the depth of cut.

Based on the formula (5), where $d_{c}$ is the milling cutter diameter $\left(d_{c}=\phi 20 \mathrm{~mm}\right), a_{p}$ is the cutting depth $\left(a_{p}=2 \mathrm{~mm}\right), b$ means the defect diameter $(b=2 \mathrm{~mm}, b=4 \mathrm{~mm}, b=6 \mathrm{~mm})$, and $g$ means depth of defect $(g=0.5 \mathrm{~mm}, g=1 \mathrm{~mm}, g=1.5 \mathrm{~mm}$ ), the percentage share of the defect $k$ in the entire cross-section of the removed layer was calculated.

$$
k=\frac{b g}{d_{c} a_{p}} 100 \%
$$

Figure 9 shows the cross-section of the machined layer on which the data needed to determine the percentages were determined.

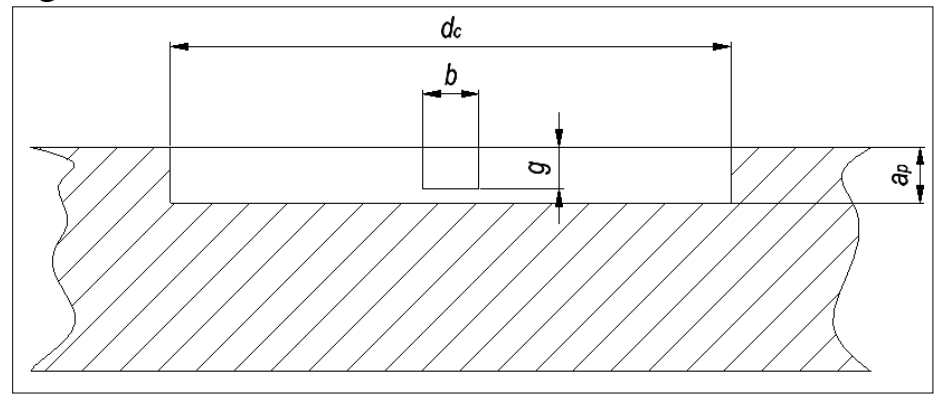

Fig. 9. Cross-section of the machined layer 
The defect with a diameter of $\phi 2 \mathrm{~mm}$, it is possible to detect a defect with a depth of not less than $1.5 \mathrm{~mm}$, which was $7.5 \%$ of the cross-sectional area of the removed layer. In addition, research were carried out on $\phi 4 \mathrm{~mm}$ and $\phi 6 \mathrm{~mm}$ diameter defects. Respectively for $\phi 4 \mathrm{~mm}$ and $\phi 6 \mathrm{~mm}$ diameter the percentages were $10 \%$ and $7.5 \%$ of the cross-sectional area of the removed layer.

Figures 10-14 show the influence of depth of the artificial defect depth on the minimum values of recurrence quantifications. The minimum values of recurrence quantifications decrease as the depth of defect increases. For defects with a diameter of $2 \mathrm{~mm}$, the indicators decrease when the defect depth is $1.5 \mathrm{~mm}$. For defects with a diameter of $4 \mathrm{~mm}$ and $6 \mathrm{~mm}$, the indicators decrease when the defect depth is $1 \mathrm{~mm}$ and $0.5 \mathrm{~mm}$, respectively.

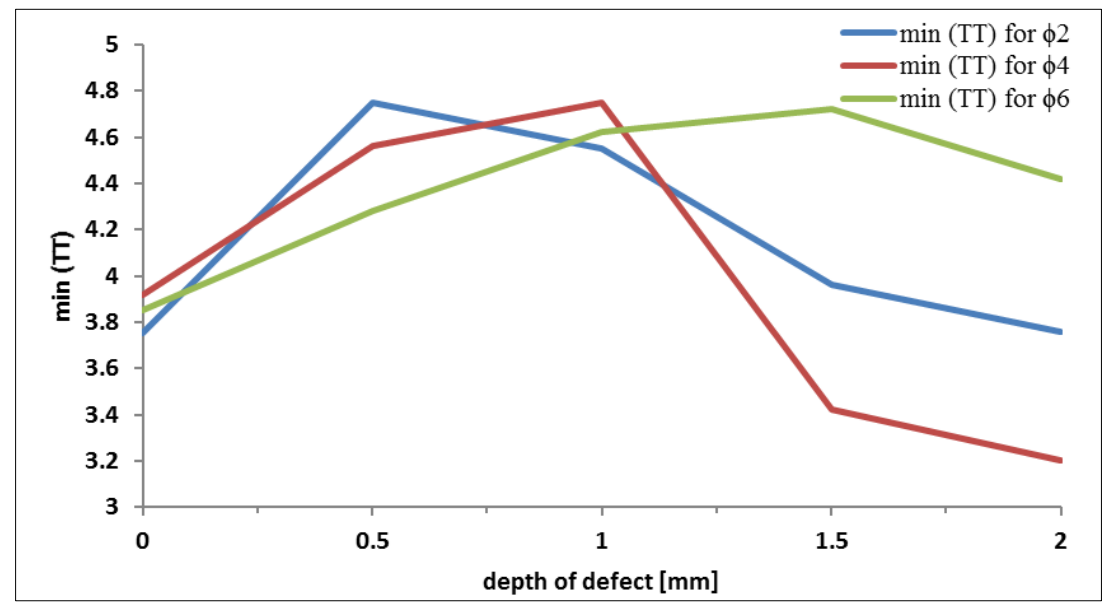

Fig. 10. Influence of defect depth on minimum values TT

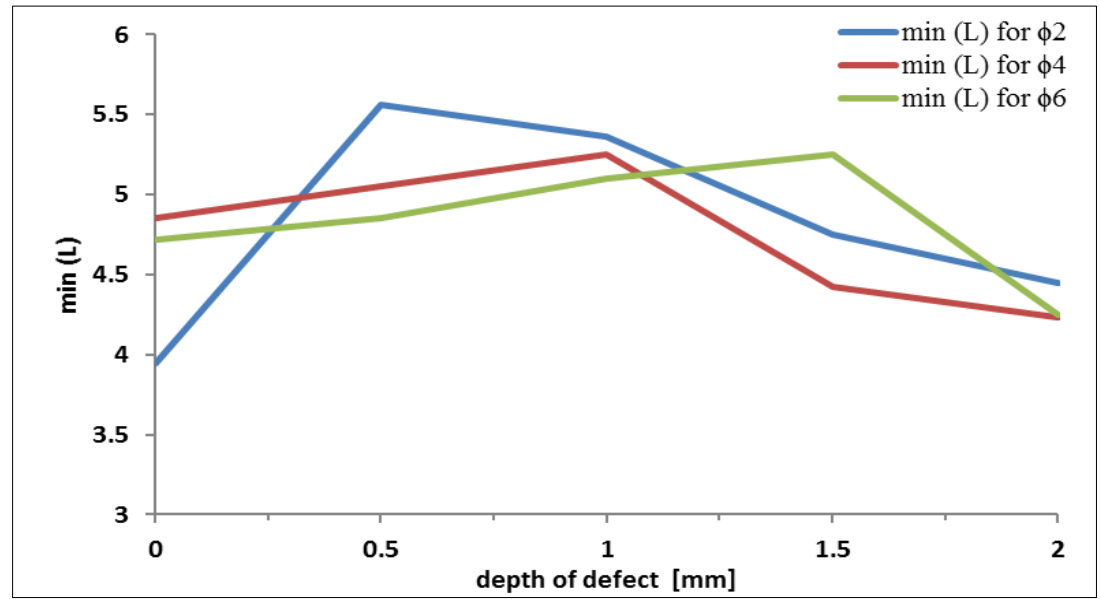

Fig. 11. Influence of defect depth on minimum values $L$ 


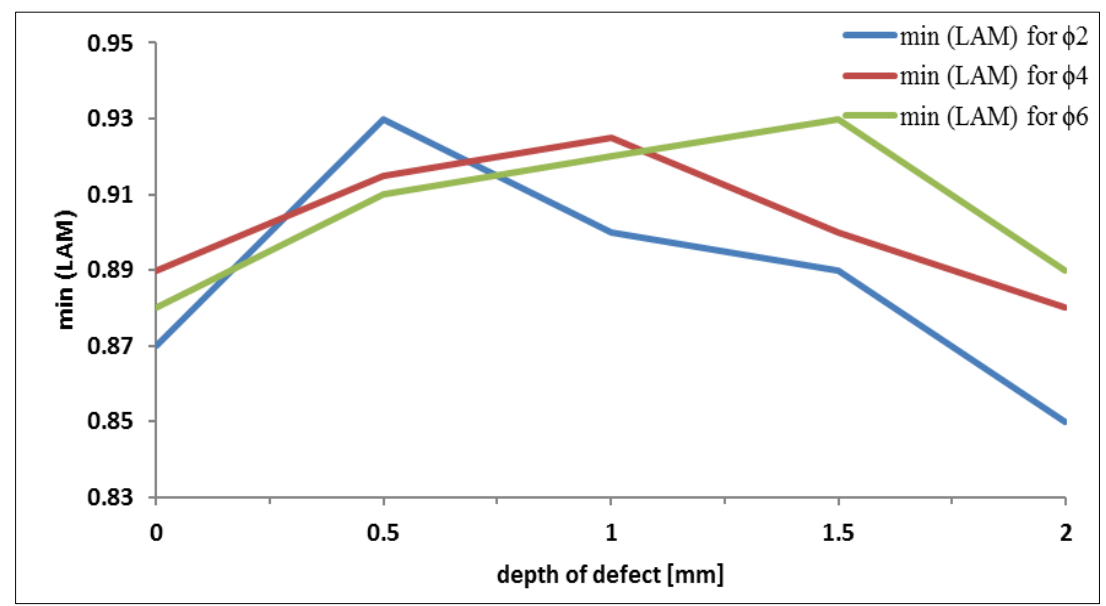

Fig. 12. Influence of defect depth on minimum values LAM

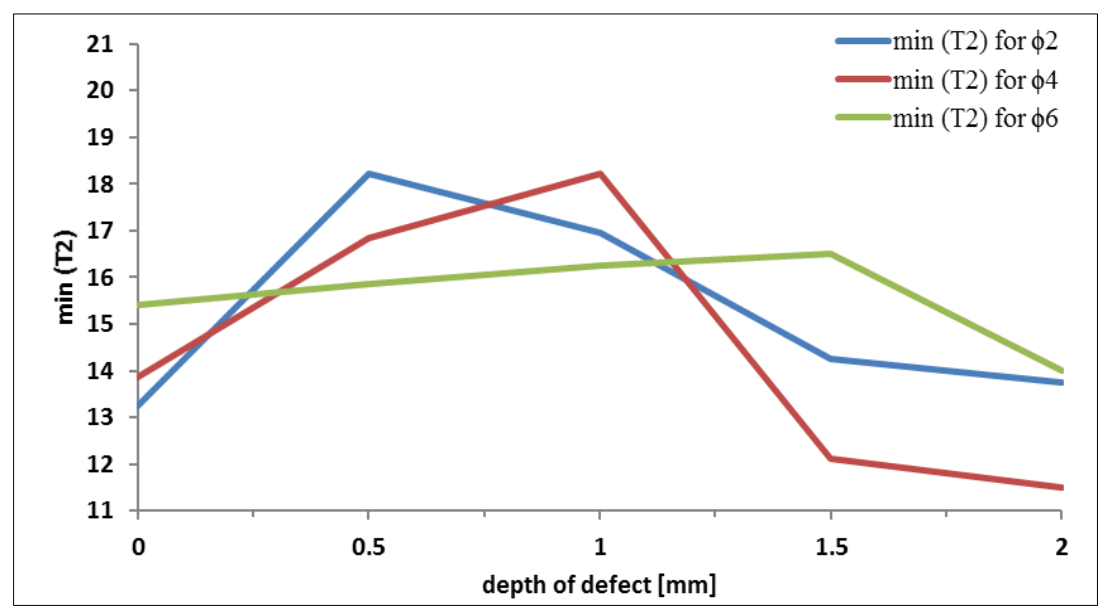

Fig. 13. Influence of defect depth on minimum values $T 2$

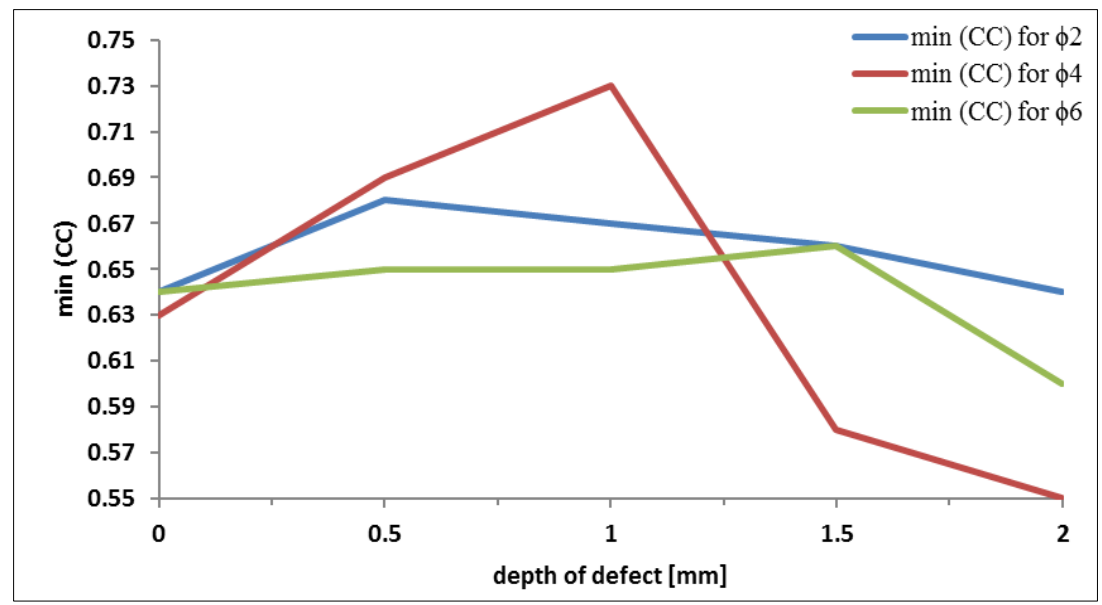

Fig. 14. Influence of defect depth on minimum values CC

Based on the obtained results it can be concluded that the recurrence indicators depend on the depth of the defect. 


\section{CONCLUSIONS}

Based on the tests carried out and the results obtained, it was found that recurrence plots (RP) and recurrence quantifications (RQA) are suitable for detecting surface defects in the form of holes at different depths with different diameters. Of the many recurrence quantifications, trapping time, average diagonal length, laminarity, recurrence time of the 2nd type and clustering coefficient are the most suitable for detecting defects.

Different types of defect depths for different diameters allowed determining the percentage share of the surface defect in the cross section of the removed layer. For defects with a diameter of $\phi 2 \mathrm{~mm}$, it is possible to detect a defect representing $7.5 \%$ of the crosssectional area of the removed layer. In turn, for a defect with a diameter of $\phi 4 \mathrm{~mm}$ and $\phi 6 \mathrm{~mm}$ it was possible to detect a defect constituting respectively $7.5 \%$ and $10 \%$ of the crosssectional area of the removed layer.

Test results and conclusions based on them allow us to assume that RP and RQA can be successfully used in other types of machining, in which the output signal is a component of the cutting force.

\section{REFERENCES}

1. Litak G., Syta A., Rusinek R., Dynamical changes during composite milling: recurrence and multiscale entropy analysis, International Journal of Advanced Manufacturing Technology 56 (2011), 445-453.

2. Kęcik K., Ciecieląg K., Zaleski K., Damage detection of composite milling process by recurrence plots and quantifications analysis, The International Journal of Advanced Manufacturing Technology 89 (2017), 133-144.

3. Kęcik K., Rusinek R., Warminski J., Stability lobes analysis of nickel superalloys milling, International Journal of Bifurcation and Chaos 21(10) (2011), 2943-2954.

4. Kęcik K., Rusinek R., Warminski J., Weremczuk A., Chatter control in the milling process of composite materials, Journal of Physics: Conference Series 382 (1) (2012), 1-6.

5. Ciecieląg K., Kęcik K., Zaleski K., Influence of defect diameter on its detection in milling process of composite material using recurrence plot technique, Composites Theory and Practice 17(4) (2017), 194-199.

6. Belaire-Franch J., Testing for Non-Linearity in an artificial financial market: a recurrence quantification approach, Journal of Economic Behavior and Organization 54(4) (2004), 483-494.

7. Marwan N., Wessel N., Meyerfeldt U., Schirdewan A., Kurths J., Recurrence Plot Based Measures of Complexity and its Application to Heart Rate Variability Data, Physics Review E 66(2) (2002), 1-8.

8. Grabowski T., Zastosowanie metody reccurence plots $\mathrm{w}$ analizie danych pomiarowych, Elektrotechnika i Elektronika 52(2) (2006), 85-96.

9. Marwan N., Kurths J., Nonlinear analysis of bivariate data with cross-recurrence plots, Physics Letter A 302 (2002), 299-307.

10. Teng, G.; Zhou, X.; Yang, C.; Zeng, X. A Nonlinear Method for Characterizing Discrete Defects in Thick Multilayer Composites, Applied Sciences 9 (1183) (2019), 1-16.

11. Eckmann J.P., Oliffson Kamphorst S., Ruelle D., Recurrence Plots of dynamical systems, Europhysics Letters 4(9) (1987), 973-977. 
12. Fabretti A., Ausloos M.: Recurrence plot and recurrence quantification analysis techniques for detecting a critical regime. examples from financial market indices, International Journal of Modern Physics C 16 (2005), 1-32.

13. Fraser A. M., Swinney H. L., Independent coordinates for strange attractors from mutual information, Physical Review A 33 (1986), 1134-4110.

14. Kennel M., Brown R., Abarbanel H., Determining embedding dimension for phase space reconstruction using a geometrical construction, Physical Review A 45(6) (1992), 3403-3411.

15. Yang Dong, Ren Wei-Xen, Hu Yi-Ding, Li Dan, Selection of optimal threshold to construct recurrence plot for structural operational vibration measurements, Journal of Sound and Vibration 349 (1992), 361-374.

16. Webber C. L. Jr, Zbilut J. P., Dynamical assessment of physiological systems and states using recurrence plot strategies, Journal of Applied Physiology 76(2) (1994), 965-973.

17. Zbilut J. P., Webber C. L. Jr, Embeddings and delays as derived from quantification of recurrence plots, Physics Letters A 171 (1992), 199-203.

18. Marwan N., Carmen Romano M., Thiel M., Kurths J., Recurrence plots for the analysis of complex systems, Physics Reports 438 (2007), 237-329.

19. Marwan N., Donges J. F., Zou Y., Donner R. V., Kurths J., Complex network approach for recurrence analysis of time series, Physics Letter A 373(46) (2009), 4246-4254.

20. Marwan N., Kurths J., Foerster S., Analysing spatially extended high-dimensional dynamics by reccurence plots, Physics Letters A 379 (2014), 894-900.

21. Gao J., Cai H. On the structures and quantification of recurrence plots, Physics Letter A 270 (2000), 75-87.

22. Wang Z., Yang C. L., Zhou X. J., Teng Y. H., Identification of localized void defects in composite by recurrence quantification analysis of ultrasonic backscattered signal, Russian Journal of Nondestructive Testing 55, 3 (2019), 192-201.

23. Brandt C., Recurrence quantification analysis as an approach for ultrasonic testing of porous carbon fiber reinforced polymers, in Recurrence Plots and Their Quantifications: Expanding Horizons, Springer International Publisher, 2016.

24. He Y., Qing H., Zhang Sh., Wang D., Zhu Sh., The cutting force and defect analysis in milling of carbon fiber-reinforced polymer (CFRP) composite, The International Journal of Advanced Manufacturing Technology, 93 (2017), 1829-1842. 